\title{
Sustained long-term clinical and radiological response with sunitinib for metastatic renal-cell carcinoma (RCC)
}

\author{
K. I. Quintyne ${ }^{1,2,3^{*}}$, T. Neenan ${ }^{1}$, F. Wallis ${ }^{4}$, R. K. Gupta ${ }^{1,5}$ \\ ${ }^{1}$ Department of Medical Oncology, Mid-Western Regional Hospital, Limerick, Ireland; \\ *Corresponding Author: keithi.quintyne@hse.ie \\ ${ }^{2}$ Graduate Entry Medical School, University of Limerick, Limerick, Ireland \\ ${ }^{3}$ School of Public Health, University of Manchester, Manchester, UK \\ ${ }^{4}$ Department of Radiology, Mid-Western Regional Hospital, Limerick, Ireland \\ ${ }^{5}$ Stokes Institute, University of Limerick, Limerick, Ireland
}

Received 25 January 2013; revised 28 February 2013; accepted 5 March 2013

\begin{abstract}
The authors herein report the case of a 67-yearold woman with metastatic renal-cell carcinoma (RCC), who has had a sustained clinical and stable radiological response to long-term therapy with an oral multi-targeted tyrosine kinase inhibitor (TKI), sunitinib with minimal lasting toxicity.
\end{abstract}

Keywords: Renal Cell Carcinoma; Biological Therapy; Sunitinib; Long Term Use

\section{INTRODUCTION}

In recent years, the incidence of renal-cell carcinoma (RCC) has gradually risen, which has been reflected with increasing cancer-related mortality data seen in Western Europe [1]. This in some aspects has been related to the paucity of available systemic therapeutic options, consequently no standard chemotherapy regimens are established. Through the advent of personalised medicine and molecular targeting, biological therapy with tyrosine kinase inhibitors (TKI), particularly sunitinib have gained significant prominence. The pivotal phase III work by Motzer et al. in 2007, which showed that the multi-targeted TKI sunitinib was associated with better quality of life and tolerability with an improved progression-free survival (PFS) of 11 months and an objective response rate (ORR) of $31 \%$, as compared to interferon alfa, at 5 months and $6 \%$ respectively [2]. No significant data is available on optimum duration of exposure to this agent, but the median duration of therapy quoted in the literature was 11 months [3].

We herein report the case of a Caucasian woman of Irish extraction who presented with metastatic RCC and proceeded with biological therapy with the oral TKI sunitinib and has been maintained on this therapy for over 38 months with minimal toxicity.

\section{CASE PRESENTATION}

A retired teacher in her late 60s, who is a lifetime nonsmoker and non-drinker, with no family history of malignancy, presented with a short history of fatigue, nonproductive cough and progressive shortness of breath. Screening plain chest radiograph was undertaken and revealed a mass adjacent to the right hilum. She underwent further investigations including bronchoscopy, which revealed no endobronchial lesions. Radiological guided biopsy was performed and histology was consistent with metastatic clear cell RCC. Computed tomography (CT) staging of his thorax, abdomen and pelvis revealed several focal lung lesions, all in the right lung, with the largest measuring $4 \mathrm{~cm}$, and a $9 \mathrm{~cm}$ malignant appearing lesion on the right kidney and no evidence of other systemic disease (see Figures 1-3).

Analysis of prognostic risk factors by the Heng criteria [4], revealed a corrected calcium level of $2.30 \mathrm{mmol} / \mathrm{L}$, haemoglobin of $11.5 \mathrm{~g} / \mathrm{dl}$, lactate dehydrogenase (LDH) of $198 \mathrm{U} / \mathrm{L}$, an ECOG performance status of 1 (Karnofsky performance status $70 \%-80 \%$ ) and a disease-free interval of $<12$ months. In November 2009, the patient was considered to be at intermediate risk, due to her performance status and the disease-free interval, and was commenced on sunitinib therapy at a dose of $50 \mathrm{mg} /$ day by schedule $4 / 2$ (6-week cycles of 4 weeks on treatment followed by 2 weeks off treatment).

She has achieved stable disease (SD) on radiological assessment for greater than 38 months, and with no evidence of new disseminated disease, demonstrated by serial restaging CTs. Toxicities reported for this patient 


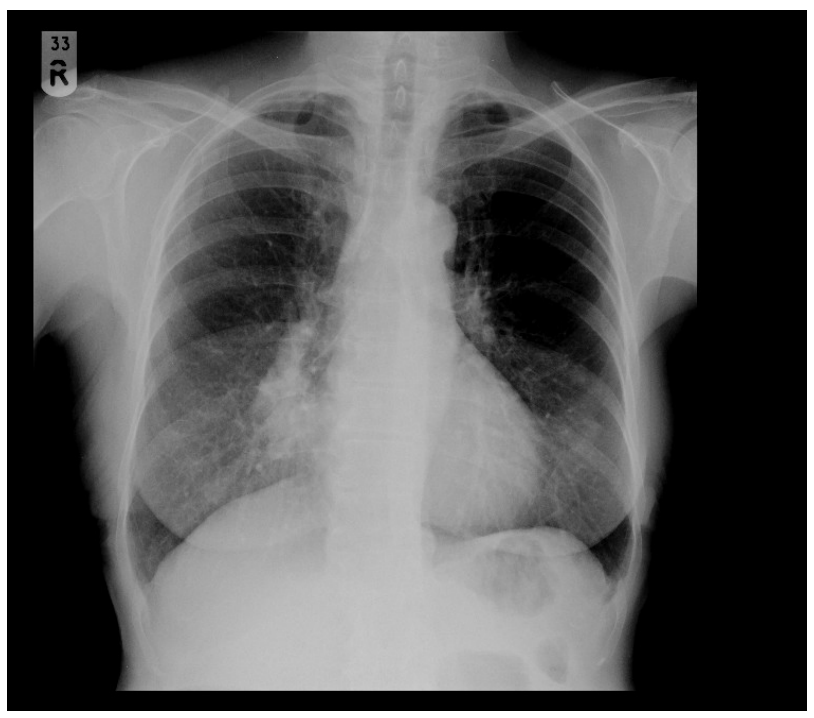

Figure 1. Plain chest radiograph at baseline.

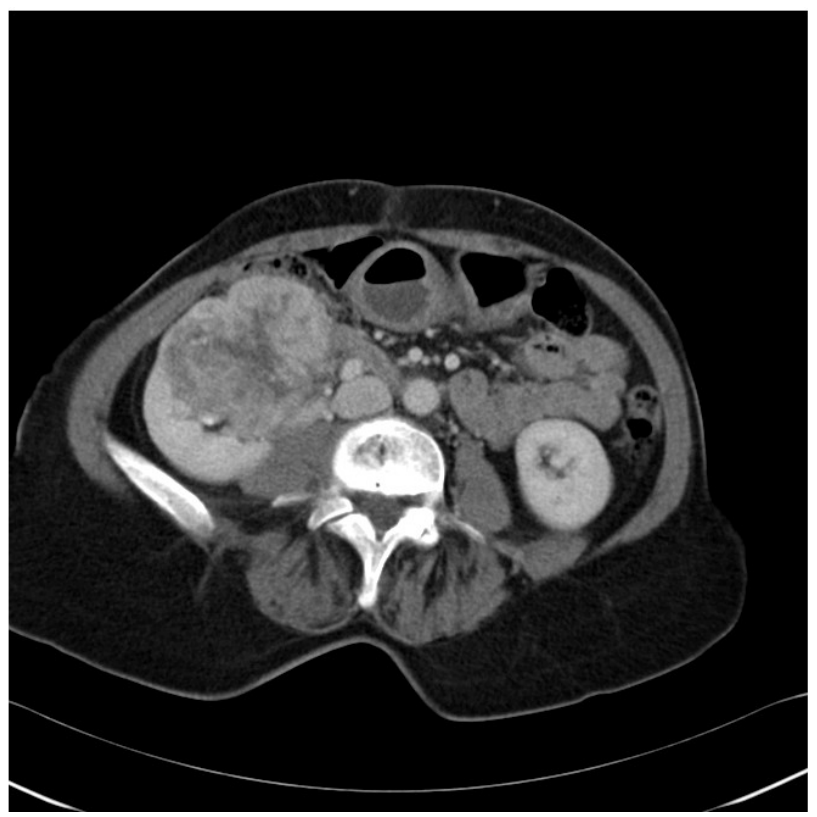

Figure 2. Staging computed tomography of abdomen at baseline.

during sunitinib therapy included hypothyroidism, one episode of grade 3 hand-foot syndrome, and weight loss $(<10 \%)$. These toxicities were managed respectively with the initiation of thyroxine therapy, topical emollient therapy and reducing the sunitinib dose level to $37.5 \mathrm{mg} /$ day (still on schedule 4/2), which allowed for the continuation of her therapy without sustained side effects or progression of her disease.

\section{OUTCOME AND FOLLOW-UP}

The patient continues to tolerate her therapy without new side effects for over 38 months. Ultimately, her qua-

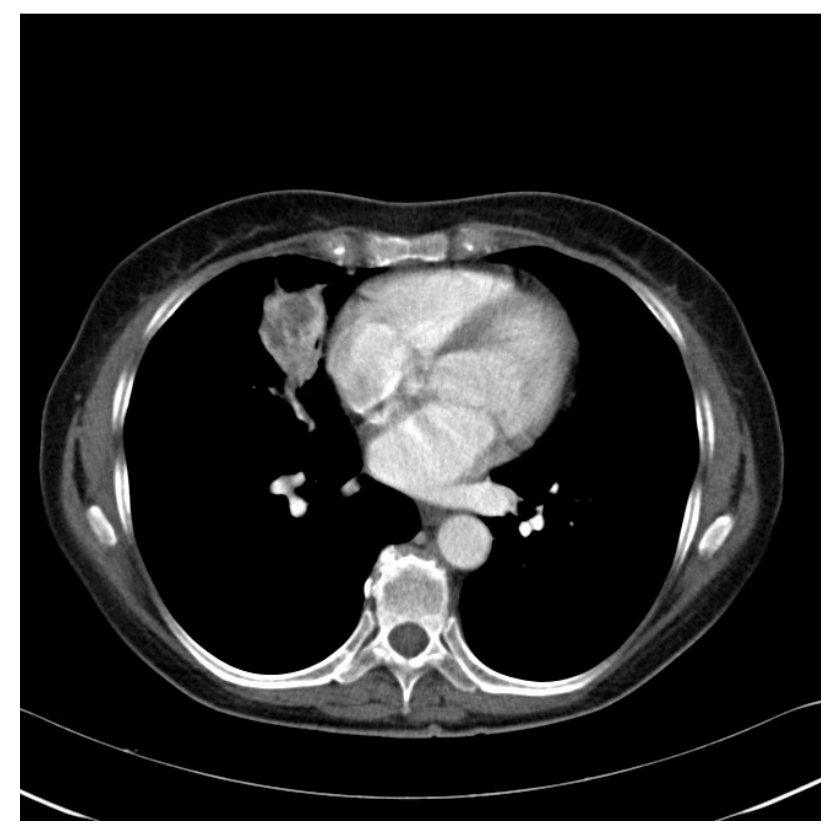

Figure 3. Staging computed tomography of thorax at baseline.

lity of life has improved as her fatigue, weight loss and respiratory symptoms have regressed and she attends her outpatient appointments and maintains a full and active social life.

\section{LEARNING POINTS}

- Patient has clinical, histological and radiological features of metastatic RCC;

- Good candidate for targeted therapy with TKI without significant lasting toxicity;

- Exceeded expected median overall survival for patient with metastatic RCC of 20 - 24 months;

- Demonstrates that patients with advanced disease can have sustained clinical benefits, once prompt and effective adverse event management is undertaken.

\section{DISCUSSION}

Metastatic RCC has been traditionally associated with a poor prognosis [5], and it has been identified as a rising cause of cancer-related deaths within the developed world [1], The most significant therapeutic manoeuvre available for many years, which offered the best outcome, was surgical resection $[1,6]$. However one-quarter of the patients with RCC present with either locally advanced or metastatic disease, and one-third of patients who have undergone radical nephrectomy for non-metastatic will develop recurrent disease [6].

Unfortunately, traditional systemic options such as chemotherapy and immunotherapy were the most appropriate modalities, but are both notoriously associated with low response rates and generally high toxicity [5,7]. RCC 
has been shown to be particularly chemotherapy-resistant with an objective response rates (ORR) of 4\% - 6\% [8]. The postulated reason for the poor ORR may be due to the expression of multidrug resistance transporter in proximal-tubule cells, from which clear cell RCC originates [6]. Immunotherapy was another option, but the median duration of response to therapy was usually 6 months [6], and often did significantly alter the natural history of the disease for most patients and was associated with high adverse reactions [5].

However, the pharmacological landscape has exploded as advances have been made in our understanding of cancer biology, with therapeutic strategies that target recaptor tyrosine kinases (RTKs). Several agents, including sunitinib has become available: this oral multitarget RTK has shown both antitumour and antiangiogenic activity [9]. It exerts its therapeutic effect by inhibiting phosphorylation of RTK, through inhibition of platelet-derived growth factor receptors (PDGF $\alpha$ and $\mathrm{PDGF} \beta$ ), vascular endothelial growth factor receptors (VEGFR1, VEGFR2 and VEGFR3), stem cell factor receptor (KIT), Fms-like tyrosine kinase-3 (FLT-3), colony stimulating factor receptor 9CSF-1R), and the glial cell-line derived neurotrophic factor receptor (RET) [10].

In the pivotal phase III multicentre randomized trial, 750 patients with advanced RCC, were offered either sunitinib (at an oral dose of $50 \mathrm{mg} /$ daily for 4 weeks, followed by 2 weeks without therapy) or interferon alfa (at a subcutaneous dose of 9 MU given thrice/weekly), and this showed a progression-free survival (PFS) of 11 months versus 5 months and ORR of 31\% versus 6\% for both agents respectively [2]. These findings were further updated by Motzer et al. and showed the median overall survival of 26.4 months and 21.4 months for sunitinib and interferon alfa respectively, with median duration of exposure of 11 months for patients receiving sunitinib [3]. As seen in own our patient the duration of exposure is presently 36 months thus far. Within the Motzer et al. updated cohort information; it was shown that $40 \%$ of 375 patients receiving sunitinib had stable disease by Response Evaluation Criteria in Solid Tumours (RECIST) [3]. Further analysis revealed that the ORR was extended across all clinical prognostic subgroups studied $[3,10]$.

The patient described here has tolerated her sunitinib therapy well, as demonstrated by her long-term use. Although the higher suggested dose could not be maintained due to dermatological toxicity experienced, given in the context that $50 \%$ of patients in the original study required dose modifications [3], this is an acceptable compromise. Her development of endocrine complications from her RTK was experienced by $14 \%$ of patients undergoing sunitinib therapy [3,6].

Based on the Motzer et al data, sunitinib has been incorporated in the treatment algorithms for patients with advanced RCC, and hence provides a much-required outpatient based therapeutic option in the armamentarium for the treatment of patients with this disease $[1,2]$.

\section{REFERENCES}

[1] Escudier, B., Eisen, T., Porta, C., et al. (2012) Renal cell carcinoma: ESMO clinical practice guidelines for diagnosis, treatment and follow-up. Annals of Oncology, 23, vii65-vii71. doi:10.1093/annonc/mds227

[2] Motzer, R.J., Hutson, T.E., Tomczak, P., et al. (2007) Sunitinib versus interferon alfa in metastatic renal-cell carcinoma. New England Journal of Medicine, 356, 115124. doi:10.1056/NEJMoa065044

[3] Motzer, R.J., Hutson, T.E., Tomczak, P., et al. (2009) Overall survival and updated results for sunitinib compared with interferon alfa in patients with metastatic renal cell carcinoma. Journal of Clinical Oncology, 27, 35843590. doi:10.1200/JCO.2008.20.1293

[4] Heng, D.X., Xie, W., Regan, M.M., et al. (2009) Prognostic factors for overall survival in patients with metastatic renal cell carcinoma treated with vascular endothelial growth factor-targeted agents: Results from a large, multicenter study. Journal of Clinical Oncology, 27, 57945799. doi:10.1200/JCO.2008.21.4809

[5] Molina, A.M., Ginsberg, M.S. and Motzer, R.J. (2011) Long-term response with everolimus for metastatic renal cell carcinoma refractory to sunitinib. Medical Oncology, 28, 1527-1529. doi:10.1007/s12032-010-9640-y

[6] Cohen, H.T. and McGovern, F.J. (2005) Renal-cell carcinoma. New England Journal of Medicine, 353, $2477-$ 2490. doi:10.1056/NEJMra043172

[7] Motzer, R.J. (2003) Renal cell carcinoma: A priority malignancy for development and study of novel therapies. Journal of Clinical Oncology, 21, 1193-1194. doi:10.1200/JCO.2003.12.072

[8] Yagoda, A., Abi-Rached, B. and Petrylak, D. (1995) Chemotherapy for advanced renal-cell carcinoma: 1983-1993. Seminars in Oncology, 22, 42-60.

[9] Schoffski, P., Bukowski, R., Flodgren, P., et al. (2009) Tyrosine kinase inhibition in renal cell carcinoma and gastrointestinal stromal tumours: Case reports. Annals of Oncology, 20, i25-i30. doi:10.1093/annonc/mdp076

[10] http://www.bccancer.bc.ca/NR/rdonlyres/EE7AE153-718 A-4AB3-A01F-66D6E49A8DC1/53411/sunitinibmonogr aph_1October2011.pdf (Accessed 12 November 2012) 